\title{
HIV risk behaviour among men who have sex with men (MSM) who attended the sexually transmitted diseases (STD) clinic, Ragama
}

\author{
Ranatunga $\mathbf{J}^{1}$, Karawita $\mathbf{D A}^{2}$, Batagalla $\mathbf{P S K}^{3}$, Senevirathne $\mathbf{M R}^{4}$, Perera WPP ${ }^{4}$, \\ Thilakaratne PTMIS ${ }^{4}$
}

\section{Key words}

Sexual risk, HIV, MSM, gay, Sri Lanka

\section{Abstract \\ Introduction}

Sri Lanka remains a low HIV prevalent country with an estimated sero-prevalence of less than $0.1 \%$ in the general population. The seroprevalence among key populations such as female sex workers (FSW), men who have sex with men (MSM), people who inject drugs (PWID) and beach boys is also $<1 \%$.(1) MSM were identified as one of the priority target groups for interventions because of their risky sexual behaviors and the increasing sero-prevalance observed among the group in the recent years.

\section{Objective}

To ascertain the level of HIV risk behaviours among MSM who attended the STD clinic, Ragama and develop necessary interventions to reduce the potential for HIV infection among them.

\section{Method}

A descriptive cross sectional study was carried out among the MSM's attending Ragama STD clinic over a period of three and a half years. MSM register and the patients records maintained at the STD clinic were used to extract the data for this study. There were 160 newly registered MSMs from January 2009 to July 2012 and all consecutive newly registered MSM were included in the sample. Data were analyzed by using the statistical package for the social sciences version 16 (SPSS v16).

\section{Results}

Majority of MSMwere in their twenties (58\%). Their level of education was relatively high and about $81 \%$ had Ordinary Level or higher qualifications. About $73 \%$ of them were engaged in some occupation while $4.4 \%$ admitted to commercial sex work as their occupation. With regard to the behavioural factors, half of them were bisexuals, and rate of insertive and receptive anal sex were $60 \%$ and $42 \%$ respectively at their most recent sex. Majority of them had sex with casual or non-regular partners (62.4\%). Level of condom use was not satisfactory. The condom use at last sex was $13 \%$ and consistent use with regular partners was $6.3 \%$. Consistent condom use during anal sex was as low as $5.7 \%$. Risk of HIV was further aggravated by the presence of STIs and use of recreational drugs. Half $(50 \%)$ were found to be positive for STIs and out of which $20 \%$ had multiple diagnoses. Fortunately, there were no injecting drug users in this sample but they had used alcohol $(30 \%)$ and smoked heroin (12\%).

\section{Conclusions}

MSM who attended the STD clinic, Ragama during the four years under study were a fairly literate community and about $68 \%$ of them were less than 30 years of age. Half of the MSM were bisexuals. Rate of insertive anal sex and receptive anal sex at

${ }^{1}$ Consultant Venereologist, STD Clinic, Colombo North Teaching Hospital, Ragama, ${ }^{2}$ Consultant Venereologist, ${ }^{3}$ Diploma Trainee in Venereology, National STD/AIDS Control Programme, ${ }^{4}$ Medical Officer, STD Clinic, Colombo North Teaching Hospital, Ragama 
last sex were $60 \%$ and $42 \%$ respectively while consistent condom use was $5.7 \%$ which constitutes a considerable risk and this risk was further aggravated by the prevalence of STIs (50\%), alcohol use $(30 \%)$ and heroin use (12\%). Therefore, risk factors for HIV transmission should be explored among MSM while maintaining good rapport and encouraging high rate of clinic attendance in order to promote protective behaviours through STI consultation and counseling.

\section{Introduction}

Among the most at risk groups, men who have sex with men are an important group to address because of their risky sexual behaviors. MSMs are defined as a group of men who have sex with other men, and thereby may transmit or acquire HIV or other STDs, but who may not necessarily share the same sexual orientation, sexual identity, or gender identity.(2) MSMs can act as carriers of STI/HIV within the general population with whom they have risk behaviors. Male-to-male sexual contact has been an important route of HIV-1 infection since HIV/AIDS was first identified nearly 30 years ago. In the past few years, there has been increased concern about newly identified and resurging epidemics of HIV infection in men who have sex with men on a global level. $(3,4)$ Against the backdrop of low and declining adult HIV prevalence in most countries, MSM continue to be disproportionately affected by HIV infection.(3) In Asia, MSM have 18.7 times the odds of being HIV infected compared with someone in the general adult population.(3) In recent years, scattered epidemiological research has identified high HIV prevalence among MSM in several Asian countries, with varying degrees of study findings and conclusions across countries, $4.5 \%$ in Cambodia; $6.4 \%$ in India; $5.2 \%$ in Indonesia and $7.1 \%$ in Malaysia. $(6,7)$ In comparison Sri Lanka has a lower prevalence of $0.9 \%$ in the region.(1)

Over the past years, it has been observed that sexually transmitted infections, especially, prevalence of syphilis is increasing among MSMs all over the world. Research among MSM in low and middle income countries has been limited due to the result of criminalization and social stigmatization of these behaviors. The safety considerations for study participants and the hidden nature of these populations are frequently faced problems.(5) Because MSM are a hidden population, they are generally under represented in population-based surveys. Available evidence suggests that social, economic or legal factors as well as individual level risk factors are likely to play important roles in levels of HIV risk taking behaviour, treatment and care options for this group of men.(8) As a significant proportion of MSM are bisexuals, the risk of transmitting the infection to the general population is high. Other risk taking behaviours among MSM such as injecting drug use and sex work puts them at more risk. All these factors need to be studied in detail to implement targeted interventions for this population.

This study is focused on the socio-demographical aspects and the identification of risk behaviors among MSM's which increases their susceptibility to HIV transmission. Counselling for consistent condom use and safe sexual behavior, outreach for delivery of services, increasing clinic attendance, regular follow up, getting the services of peers for knowledge dissemination, condom promotion and regular screening for STI/HIV are the interventions planned based on the research findings.

\section{Method}

The study was carried out at the Ragama STD clinic which is situated in the Colombo North Teaching Hospital, at Ragama. Teaching Hospital, Ragama, one of the major tertiary care institutions in Sri Lanka is situated in the district of Gampaha which is adjacent to the Colombo district. The STD clinic, Ragama manages around 2500-3000 patient visits per year and another 3000-3500 visits for other services. Number of new patients seen per year is about 700 800.

A descriptive cross sectional study was carried out among the MSMs attending Ragama STD clinic over a period of three and a half years. MSMs register 
and the patient records maintained at the STD clinic were used to extract the data for this study. There were 160 newly registered MSMs from January 2009 to July 2012 and all consecutive newly registered MSMs were taken into the sample. There were 39 MSMs in 2009, 38 in 2010, 47 in 2011 and 36 in 2012. Data were analyzed by using the statistical package for the social sciences version 16 (SPSS v16). Ethical approval for the study was obtained from the ethical review committee of the Faculty of Medicine, University of Kelaniya.

\section{Results}

\section{Demographic Characteristics}

Majority of MSM were in their twenties $92(58 \%)$ while $16(10 \%)$ were below the age of 20 years, 42 $(26 \%)$ were in their thirties, and only10 (6\%) were above the age of 30 years. Their level of education was relatively high and $98(81 \%)$ had passed the Ordinary Level examination or had higher qualifications. $114(73 \%)$ of them were en gaged in occupation (Figure1) while 7 (4.4\%) admitted to commercial sex work as their occupation; others were unemployed 35 (21.9\%).

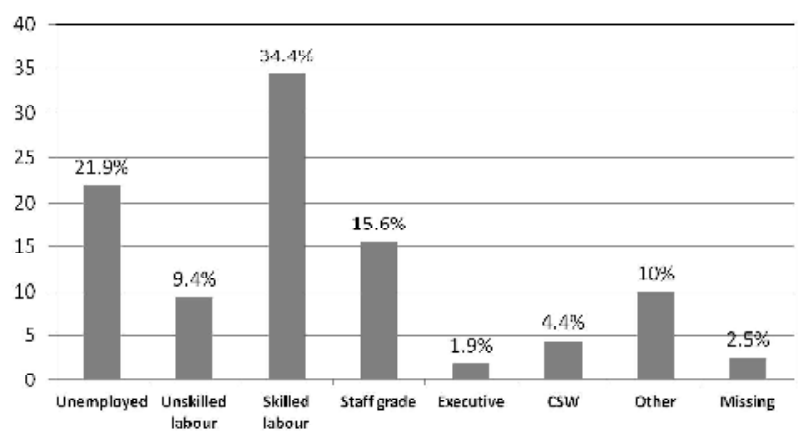

Figure 1: Categories of occupation in the sample

\section{Behavioural Characteristics}

Half of the sample (79) were bisexuals, and the rates of insertive and receptive anal sex were $60 \%$ and $42 \%$ respectively at their most recent sex. Majority of them, 66 (41.5\%) had sex with casual partners. Condom use at last sex was $13 \%$ and consistent use with regular partners was $6.3 \%$ (Figure 2). Consistent condom use with anal sex was as low as $5.7 \%$ Risk of HIV was further aggravated by the presence of STIs and use of recreational drugs. Half of them $(50 \%)$ were found to be positive for STIs (Figure 3) and out of which $20 \%$ had multiple diagnoses. Among the STIs, syphilis was the most common infection $(33.5 \%)$ and about two third of them were in the infectious stages. The next common infection was human papilloma virus infection (11.2\%) followed by genital herpes (7.9\%). Prevalence of gonorrhoea (3.3\%) was higher than non gonococcal urethritis (2.6\%). The prevalence of HIV among this population was $1.3 \%$. About $40 \%$ were diagnosed with non-venereal diseases. There were no injecting drug users in this sample but they used alcohol (30\%) and smoked heroin (12\%).

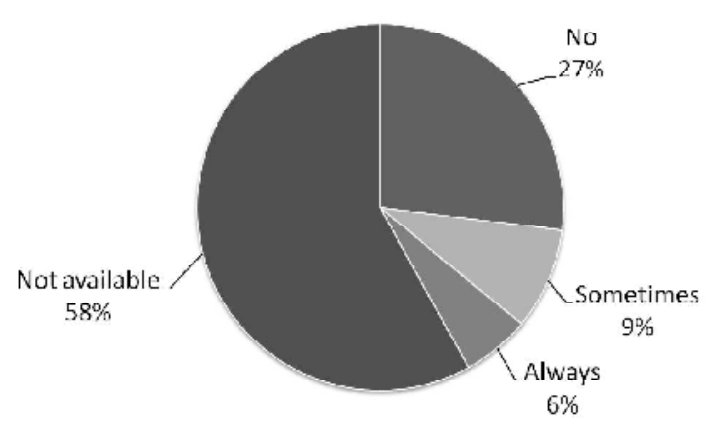

Figure 2 : Condom use with regular partners

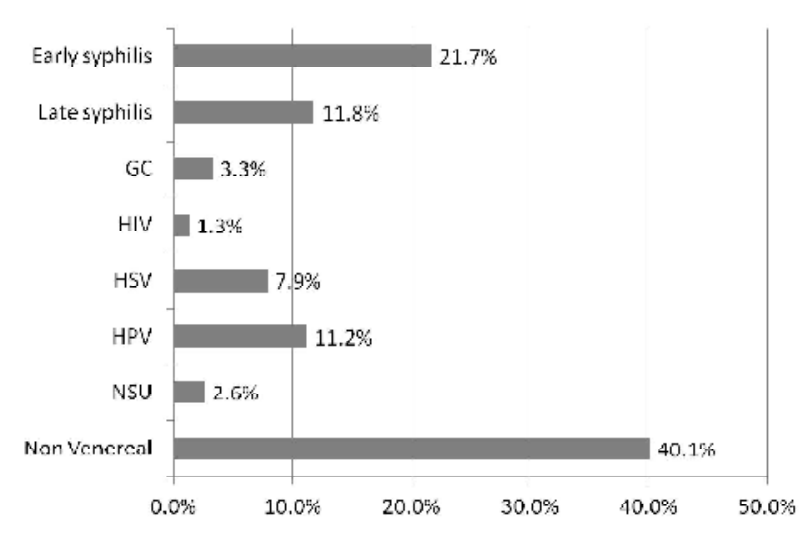

Figure 3: Prevalence of STI's among MSM 


\section{Discussion}

MSM's who attended the STD clinic, Ragama during the previous four years were a fairly literate community and about $68 \%$ of them were less than 30 years of age. Half of the MSM's were bisexuals. Therefore, they can be carriers of infections to other populations with whom they have unprotected sex. Therefore they act as a bridging population between the most atrisk populations and the general population. This is a well identified mode of HIV spread into the general population in most of the countries. In a study done to assess the risk factors among MSM in low and middle income countries, the lifetime frequency of ever had sex with a woman was 50\%.(10)

Rate of insertive anal sex and receptive anal sex at their last sex were high while consistent condom use was very low, which is a considerable risk for acquiring and transmitting sexually transmitted infections. As this sample was taken from MSM who attended a health care facility, they may not represent the MSM population in the country. But the fact that their literacy rate being fairly good, leaves us with a question why they didn't practice safe sex?

Available data shows very low rate of condom use with the regular partner and this reflects the low risk perception with regular partners. Unavailability of condoms at the time of need, perceived or actual lack of pleasure with condoms, lack of knowledge, inability to keep condoms with them or lack of adequate knowledge may have contributed for low condom use. In a study done in New York city, to compare the syphilis and HIV infected MSM with heterosexuals, MSM differed significantly on all demographics and reported a higher prevalence of condom use at last sex $(62.9 \%$ vs. $38.3 \%)$ and of past-year HIV testing (53.6\% vs. $27.2 \%$ ) but also more past-year sex partners.(9) HIV and syphilis rates were 2526.9/ 100,000 and 707.0/100,000, each of which was over 140 times when compared with heterosexuals. The hidden nature and discriminatory attitudes towards same sex practices in Sri Lankan culture may be a contributory factor for low condom use and low uptake of HIV testing. In this study, the prevalence of HIV among the MSMs recruited to the study was $1.3 \%$ and this is higher than the national figure, which is $0.9 \%$. Sri Lanka still reports a low prevalent situation despite the fact that the prevalence among MSM have risen from $0.48 \%$ in 2009 to $0.9 \%$ in 2011.(1) This may be due to an actual increase in the prevalence among a cluster in Gampaha district or due to the national figure being calculated during sentinel surveillance using the national estimate of MSMs $(40,000)$ as the denominator. This was calculated from a sample who attended a health care facility as they perceived some risk or with symptoms. Risk of acquiring and transmission of $\mathrm{HIV}$ is further aggravated by the prevalence of STIs which was very high $(50 \%)$ in this sample. Early syphilis was the highest reported while gonorrhea also was significantly high. Again this figure cannot be generalized, because we have taken the sample from a health care facility. Sexually transmitted infections like syphilis, gonorrhoea and non gonococcal urethritis reflects unprotected sex in the recent past. This should be taken as an early warning for new HIV infections. As HIV will present later, this figure itself shows the need for early targeted interventions for this population. In a study done in Bangkok, Thailand using a sample from a community centre, to assess the prevalence of Treponema pallidum (TP) seropositivity and herpes simplex virus type 2 (HSV2) infection and risk factors associated with their prevalence in a cohort of MSM with baseline behavioural data and laboratory testing for sexually transmitted infections (STIs) showed that from a total of 1544 enrolled men (mean age 26 years) TP, HSV2 and HIV seropositive rates were $4.4 \%, 20.7 \%$ and $21.6 \%$, respectively. Participating in group sex, reporting paying for sex, reporting sex with a casual partner in a park and being HSV-2 seropositive were associated with TP prevalence. Age around 30 years, having less than a high school education, past use of recreational drugs, meeting casual sexual partners at a public venue (sauna) and TP seropositivity were associated with HSV-2 infection. The significant baseline prevalence of TP seropositivity and HSV-2 infection in this cohort demonstrates the need for screening and treatment of these STIs and the 
importance of targeted interventions.(11) In this study, the syphilis seropositivity was $33.5 \%$. As this was a retrospective study, we could analyze only the available data and HSV seropositivity was not available. Further, alcohol use (30\%) and heroin use (12\%) also will put them at risk of acquiring HIV. Although there were no injecting drug users in this sample, adequate surveillance, size estimation and geographical mapping is needed to prevent a future epidemic driven by drug users. Therefore, risk factors for HIV transmission should be explored among MSMs while keeping a good relationship by making the clinic environment friendly for them to get regular screening and to improve contact tracing and treatment.

As secondary data were used for this study, the reasons for notusing condoms, behavior modifications after attending the clinic and difficulties encountered when seeking care and attitudes towards services were not analyzed. A prospective study should be planned in order to assess the impacts of services and stigma discrimination within the government health care facilities.

\section{Conclusions}

MSMs who attended the STD clinic, Ragama during the four years under study were a fairly literate community and about $68 \%$ of them were less than 30 years of age. Alarmingly, half of them were bisexuals posing the threat of carrying the infection to the general population. High risk behaviours like receptive and insertive anal sex was very high among this population with a low condom use which is evident of a possible concentrated epidemic among the MSMs in Sri Lanka. The high rates of highly infectious sexually transmitted infections among this population carries the risk of high HIV transmission and is an evidence for unprotected sex which further aggravates the HIV transmission among this group and to the general population. Injecting drug use was low among the studied group.

\section{Recommendations}

Targeted interventions should be planned taking the study findings into consideration. A peer led programme to escort MSMs to STD services is highly necessary at this stage. The STD clinics should be friendly for this community and staff training to minimize stigma and discrimination should be undertaken at the same time. Even though same sex activities are illegal in Sri Lanka, the mass media should be used in a culturally accepted manner to educate the risk groups as well as the general population. Detailed research should be undertaken to analyze the factors contributing to risky sexual behaviors.

\section{References}

1. Department of Health. HIV Sentinel Serosurveillance Survey. Sri Lanka; National STD/ AIDS Control Programme: August 2012.

2. Mayer KH and Carballo-Diéguez Alex. Homosexual and bisexual behaviour in men in relation to STDs and HIV infection. In: Holmes KK, Sparling PF, Stamm WE, Piot P, Wasserheit $J \mathrm{~N}$, Corey Let al. editors. Sexually Transmitted Diseases. $4^{\text {th }}$ ed. New York: McGraw Hill; 2008. p 203.

3. Baral S, Sifakis F, Cleghorn F, Beyrer C. Elevated risk for HIV infection among men who have sex with men in low- and middle-income countries 2000-2006: a systematic review. PLoS Med 2007; 4:339.

4. Van Griensven F, Van Wijngaardenc JW, Baral S, Grulich A. The global epidemic of HIV infection among men who have sex with men. Curr Op in HIV AIDS. 2009 Jul; 4(4):300-7. doi: 10.1097/COH.0b013e32832c3bb3.

5. Beyrer C, Wirtz AL, Walker D, Johns B, Sifakis F, Baral S. The Global HIV epidemic among men who have sex with men. Washington DC; World Bank Report 2011, p1-4. 
6. Baral S, Sifakis F, Masenior N, Cleghorn F, Beyrer C. A systematic review of HIV prevalence among MSM in Asia, Latin America, and Africa from 2000-2008. XVII International AIDS Conference. 2008. Abstract no. TUPE0285.

7. UNAIDS. 2008 Report on the global AIDS epidemic. Annex 1. HIV and AIDS estimates and data. Available at: http://data.unaids.org/pub/

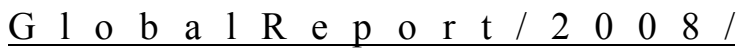
jc1510 2008 global report pp211 234 en.pdf

8. Adebajo S, Karlyn A, Enweonwu P, Tocco J. Social and economic factors contributing to the HIV vulnerability of MSM in urban Nigeria: A qualitative study comparing Lagos and Kano. XIX International AIDS Conference; Washington, DC. 22-27 July 2012.

9. Pathela P, Braunstein SL, Schillinger JA, Shepard C, Sweeney M, Blank S. Men who have sex with men have a 140-fold higher risk for newly diagnosed HIV and syphilis compared with heterosexual men in New York City: J Acquir Immune Defic Syndr. 2011 Dec 1; 58(4):408-16. doi: 10.1097/QAI.0b013e318230e1ca.

10. Cáceres CF, Konda K, Segura ER, Lyerla R. Epidemiology of male same-sex behavior and associated sexual health indicators in low- and middle-income countries: 2003-2007 estimates: Sex Transm Infect. 2008 Aug; 84 Suppl 1:49-56. doi: 10.1136/sti.2008.030569.

11. Holtz TH, Thienkrua W, McNicholl JM, Wimonsate W, Chaikummao S, Chonwattana W, Wasinrapee P, Varangrat A, Mock PA, Sirivongrangson P, van Griensven F. Prevalence of Treponema pallidium seropositivity and herpes simplex virus type 2 infection in a cohort of men who have sex with men, Bangkok, Thailand, 2006-2010: Int J STD AIDS. 2012 Jun; 23(6):4248. doi: 10.1258/ijsa.2011.011256. 